\title{
Optimal Dispatch of Ancillary services via Self-Organizing Migration Algorithm
}

\author{
Jakub Novák, Vladimír Bobál, Petr Chalupa \\ Faculty of Applied Informatics, Tomas Bata University \\ Zlin, Czech Republic (e-mail: \{jnovak, bobal, chalupa\}@fai.utb.cz)
}

\begin{abstract}
Modern electric power systems are large-scale systems with a complex structure comprised of interconnected networks. The balance between the generation and consumption of electricity has to be maintained at any moment. Transmission System Operator (TSO) uses ancillary services for keeping the domestic power balance. Selection of the services can be viewed as an optimization problem. In this contribution the optimization problem is solved via the Self-Organizing Migration Algorithm which belongs to the class of evolutionary algorithms.
\end{abstract}

Keywords: Evolutionary computations, ancillary services, frequency control, optimal dispatch

\section{INTRODUCTION}

Modern electric power systems are large-scale systems with a complex structure comprised of interconnected networks. The Union for the Coordination of Transmission of Electricity (UCTE) coordinates the operation and development of the electricity transmission grid in 24 countries, including Czech Republic as depicted in Figure 1. UCTE transmission system is divided into control areas - typically by countries, although some subsystems are joined together to create utilities that are able to operate independently in case of emergency - e.g. CENTREL. Transmission System Operator (TSO) manages the operation of the power system within the control area and coordinates its activities with neighbouring utilities. The TSO is responsible for taking care of the safe transmission of electricity, the reliability and stability of the system and balancing supply and demand at any time.

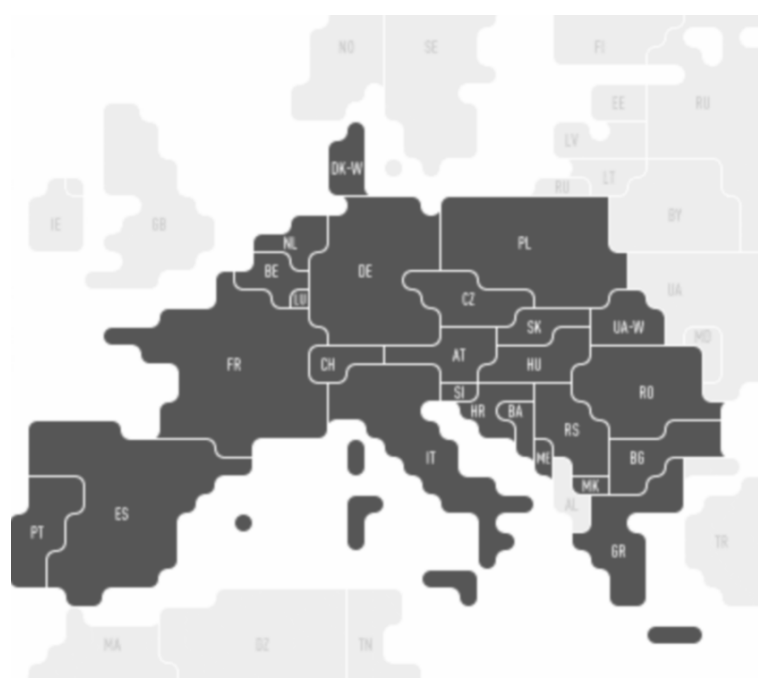

Fig. 1. Synchronously linked grid of UCTE

Since the ISO does not own any generation, it must ensure that there is sufficient unloaded capacity among on-line generators for maintaining the reliability of the system. To reduce the risk some ancillary services are obtained through long term contract and some are bought through spot market.

The provision of ancillary services is an economic and technical issue that must be addressed in all deregulated power market structures. Since TSO works on behalf the users of the system it has to decide how much ancillary services should be bought. If there are not enough services then TSO cannot ensure the security of the system. However, the costs for the AS are passed to system user.

The interdependence between the security margin, the energy schedules and the ancillary services makes the problem of choosing the optimal energy and ancillary service (AS) configuration difficult. The problem is formulated as a mixed integer, nonlinear optimization problem taking into account both continuous and discrete control variables. The objective of the reserve dispatch in a multi-area electricity market is to minimize the expenses for the reserves subject to a number of constraints.

The dispatch problem for electricity generation has been the focus of numerous studies. Arnott et al. (2003) reviewed frequency control practices in different countries, summarised experience gained from market changes and suggested ways in which frequency control needs further development. Chuvychin et al. (1996) applied an adaptive scheme which uses both frequency and rate-of-change of frequency measurements to set load shedding and technique for coordination with spinning reserve is presented. Wang et al. (2002) modified P-Q decoupled optimal power flow (OPF) to employ for the real-time optimal dispatch problem.

The power system operator is faced by many optimization problems in power system operation. Many classical optimization techniques have been applied to these problems, such as linear or quadratic programming (Fan and Zhang, 1998). These classical techniques use approximations and linearization procedures in order to limit complexity of the optimization problem. Chand and Sugianto (2005) used a 
heuristic based technique called horizon scan to find optimal solution for the problem of economic dispatch with linear and nonlinear constraints given by the dynamics of power systems. Schmidt and Verstege (2001) applied a Pareto-based evolution strategy to minimize the costs for providing reserve aid for covering transmission losses. In Abou El-Ela et al. (2005) the optimal preventive control actions are obtained via multi-objective fuzzy linear programming (MFLP) technique. Other optimization techniques such as particle swarm optimization (Yoshida et al., 2000) or genetic algorithms (Damousis et al., 2002) have been also applied for dispatch optimization.

\section{ANCILLARY SERVICES}

Power systems are subjected to sudden and unpredictable changes due to changes of generation and fluctuations of loads. Therefore continuous regulation is essential in maintaining system frequency. If generation exceeds load then frequency rises. If load exceeds generation frequency falls. Continuous regulation is also important in controlling inter-area power flows. If generation exceeds load within one balancing area, then power will flow over the transmission line ties to adjacent areas. To be able to respond to contingencies without affecting overall reliability system operators have a coordinated set of operating reserves. If there is a generator outage the frequency has to return to its preset value of $50 \mathrm{~Hz}$ within 15 minutes. The Czech TSO operator ČEPS defined following ancillary services to balance contingencies:

$\begin{array}{ll}\text { - } & \text { Primary frequency control } \\ \text { - } & \text { Secondary frequency control } \\ \text { - } & \text { Tertiary control } \\ \text { - } & \text { Quick-start } \\ \text { - } & \text { Eispatch reserve } \\ \text { - } & \text { Balancing energy } \\ \text { - } & \text { Black-start capability }\end{array}$

- Primary Frequency Control PR is an automatic response to frequency changes reacting immediately at the generation units. The primary regulation reserve RZPR must be released within 30 seconds. The primary control is Proportional control (P-control), where the output change is directly proportional to frequency deviation.

- Secondary Frequency Control SR should reset the primary control reserves in $5-15$ minutes to be ready for next disturbance. Dispatch centre computers measure ACE and send set-points for regulating power plants automatically.

- Tertiary control TR consists of a change of the operating point of the generation unit's output based on request sent to the power plant by the dispatch centre at TSO. The whole regulation reserve for tertiary control must be provided within 30 minutes at the minimum rate $2 \mathrm{MW} / \mathrm{min}$. The tertiary control is used to free up the exhausted secondary reserve so it can effectively balance the frequency fluctuations. The reserve denoted RZTR- is used to decrease the output and RZTR+ to increase the output.
- Quick-start reserve QS is a reserve that contains units capable of providing the reserves within 10 minutes. The main purpose of this reserve is the correction of power imbalances occurring as a consequence of a failure at a power plant or a significant load increase.

- Dispatch reserve DZ is provided by units that are capable of reaching nominal or agreed value within 90 minutes. These reserves are activated upon request from TSO dispatch centre. The provider must guarantee minimum 24 hours duration of provision of dispatch reserve.

- Emergency assistance HV from abroad is based on mutual agreement with neighbouring TSOs.

- Balancing energy EregZ can be purchased from abroad. The inquiry to the source TSO must be submitted by the provider 2 hours before the scheduled supply of EregZ. It is possible to activate or terminate the supply of EregZ on a change of business interval, currently on the hour. This energy is not guaranteed and may not be obtained when it is needed.

- Black-start capability is provided by the blocks that are able to start without any external source of energy.

The key point is to optimally select the appropriate AS set at lowest costs for AS reservation. Currently, an expert system, developed in Havel et al. (2008a, 2008b), helps the Czech TSO to better understand the transmission system and recommends the optimal set of AS to be purchased to meet the required reliability indices.

The expert system uses Monte-Carlo simulations for analysis of the behaviour of the grid under the different distribution of AS. The model also includes a model of a human operator at the dispatch centre who activates the AS.

\section{REAL-TIME FREQUENCY CONTROL}

Frequency control is the real-time control of all generation and transmission resources that are currently online and available to meet load and to maintain reliability within the control area. Frequency will deviate from the nominal setting whenever there is any imbalance between generation and load as the imbalance will be drawn from the kinetic energy stored in the rotating masses of the generators. ACE is a difference between the scheduled and actual foreign power exchange corrected with the effect of the primary control, which acts independently of the central controller, to avoid counter-regulation. The ACE should be kept at the zero level since negative ACE represents unscheduled export and positive ACE represents unscheduled import.

The Area Control Error (ACE) is defined in UCTE Operational Handbook (2004) as

$$
A C E={ }_{\triangle} P+K \Delta f
$$

where $\Delta \mathrm{P}[M W]$ is a power control error (measured difference between scheduled and actual interchange with neighbouring areas), $\Delta \mathrm{f}[\mathrm{Hz}]$ is a frequency error (deviation 


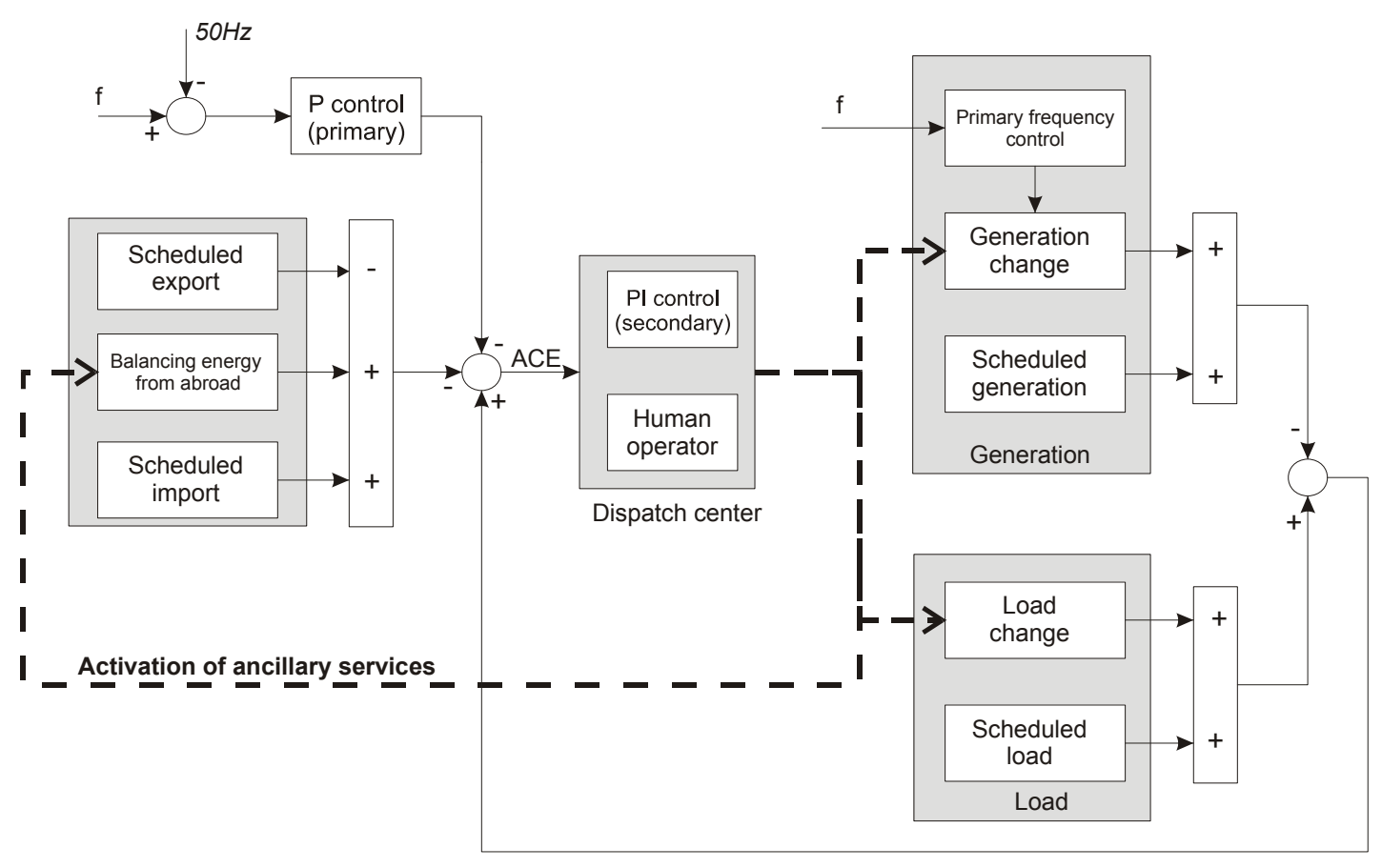

Fig. 2 Power balance control

of the measured frequency $f_{m}$ from the frequency set-point $\left.f_{S P}\right)$

$$
\Delta f=f_{m}-f_{S P},
$$

$\mathrm{K}[\mathrm{MW} / \mathrm{Hz}]$ is a frequency bias of the control area called $K-$ factor in the UCTE's terminology. The term $K \Delta f$ compensates for the action of the distributed primary control within the area in order to avoid counter-regulation from the central PI controller at the TSO's dispatch centre. This ensures that the secondary control will only be called up in the control area which is the source of the disturbance.

During real time, the TSO shall dispatch generating units, loads and system resources to procure imbalance energy as depicted in Figure 2. In addition, the TSO may also need to purchase additional AS if the services arranged in advance are used to provide imbalance energy, and such depletion needs to be recovered to meet reliability contingency requirements. TSO chooses the generating units, loads and system resources only based on price, and the effectiveness (location and ramp rate) of the resource concerned to respond to the fluctuation in generation or demand.

Dispatch centre uses a database for dispatch of ancillary services that includes

$$
\begin{array}{ll}
\text { - } & \text { generating unit name } \\
\text { - } & \text { quantity bid } \\
\text { - } & \text { price } \\
& \text { type of ancillary service provided }
\end{array}
$$

The primary control loop is the first to react for imbalances. In order to maintain system frequency at the nominal value and schedules between control areas, a secondary frequency control loop or Automatic Generation Control (AGC) coordinates the individual raise and lower signals to the generators. The control centre gathers the relevant frequency and power flow information and sends the appropriate set point adjustments for units that can provide ancillary services.

The first step of the dispatch optimization process is forecasting the $\Delta \mathrm{P}$ for the prediction horizon of 6 hours. Using the statistical data from past years, actual and past $\Delta \mathrm{P}$, daily diagram of $\Delta \mathrm{P}$ and exponential forgetting, a vector that contains 5-minutes prediction of $\Delta \mathrm{P}$ is constructed.

$$
\Delta P=[\Delta P(1), \Delta P(2), \ldots \ldots, \Delta P(72)]
$$

The overall costs that has to be minimized on the prediction horizon are given as a sum of costs for ancillary services 
$C_{A S}[C Z K]$ and costs for uncovered control error $\triangle P$ that is denoted as $C_{\triangle P}[C Z K]$.

$$
C=C_{A S}+C_{\triangle P}
$$

The costs for uncovered control error $C_{\triangle P}$ are given as

$$
C_{\Delta P}=\sum_{k=1}^{360} \Delta P(k) p_{\Delta P}
$$

where $\Delta P(k)$ is the value of uncovered control error at step $\mathrm{k}$ and $p_{\triangle P}[C Z K / M W]$ is its price. The price $p_{\triangle P}$ is assumed to be constant.

The cost for ancillary services depends on the amount of activated AS on prediction horizon.

$$
\begin{aligned}
& C_{A S}=C_{T R+}+C_{T R-}+C_{H V}+C_{D Z}+C_{Q S}+C_{E r g Z Z} \\
& C_{T R+}=\sum_{k=1}^{360} \sum_{i=1}^{n T R+} T R_{i}^{+}(k) p_{T R+}^{i}(k) \\
& C_{T R-}=\sum_{k=1}^{360} \sum_{i=1}^{n T R-} T R_{i}^{-}(k) p_{T R+}^{i}(k) \\
& C_{Q S}=\sum_{k=1}^{360} \sum_{i=1}^{n Q S} Q S_{i}(k) p_{Q S}^{i}(k) \\
& C_{D Z}=\sum_{k=1}^{360} \sum_{i=1}^{n D Z} D Z_{i}(k) p_{D Z}^{i}(k) \\
& C_{E r e g Z}=\sum_{k=1}^{360} \operatorname{EregZ}(k) p_{\text {Ereg }}(k) \\
& C_{H V}=\sum_{k=1}^{360} H V(k) p_{H V}(k)
\end{aligned}
$$

where

$T_{\text {vectors }}{ }^{+} \ldots . T R^{+}{ }_{n T R+}, T R_{i}^{-} \ldots . . T R_{n T R-}^{-}, Q S_{i} \ldots . Q S_{n Q S}$, contain the
$H V, E r e g Z, D Z[M W]$

amount of activated ancillary service for each generating block that provides ancillary services, $p_{T R+}^{i}, p_{T R-}^{i}, p_{Q S}^{i}, p_{D Z}^{i}$,
$p_{E \operatorname{reg} Z}, p_{H V}[C Z K / M W]$ are prices of these block and $n Q S, n T R+, n T R-, n D Z$ are numbers of generators that provide certain type of ancillary service. The minute prices of blocks are obtained from the hour prices which can alter during the day.

$$
p_{T R+}^{i}[C Z K / M W]=\frac{p_{T R+}^{i}[C Z K / M W h]}{60}
$$

The secondary frequency control SR is not included in cost criterion because it is an automatic service and amount of activated SR is given by the PI controller.

The optimization task is to find the minimum of the overall costs $C$ with respect to constraints. The main constraints for the optimized parameter arise from the amount of ancillary services that have been purchased in a day-ahead market or through bilateral contract. The amount of energy that can be purchased from abroad is limited by the free capacity on profiles between the national power grids. Other constraints for parameters that describe the course of AS on the future horizon are time delays, rates of growth, maximal and minimal amount of activations of each AS. E.g. Dispatch reserve which can be activated only at its nominal value has time delay of 90 minutes. It reaches the nominal value in 15 minutes and must be activated for minimal time of 2 hours. The activation of the block that provides DZ with the nominal value of $70 \mathrm{MW}$ for 3 hours at the current time instant $t$ would result in the course as depicted in Fig. 3.

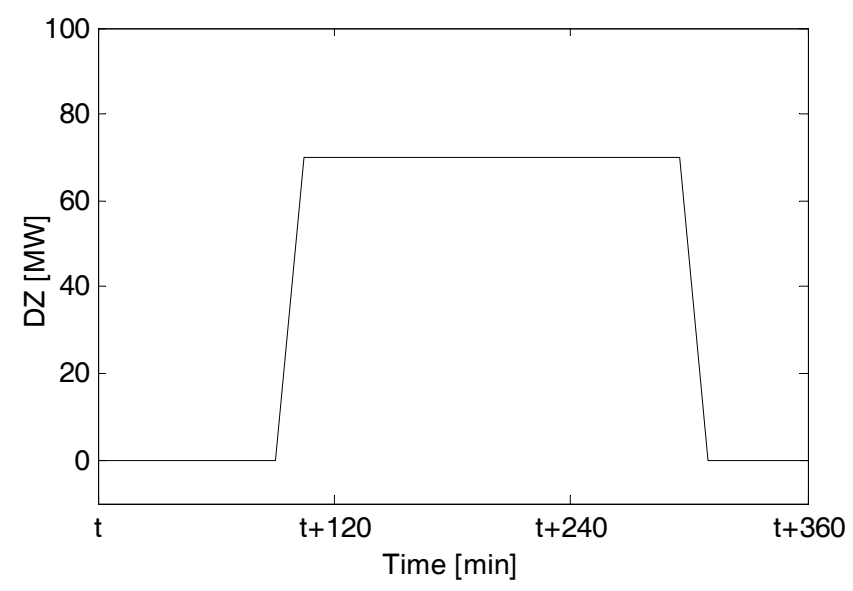

Fig. 3. Activation of Dispatch Reserve

\section{OPTIMIZATION TOOL}

Self-Organizing Migration Algorithm proposed in Babu and Onwubolu (2004) is based on cooperative searching (migrating) the area of all possible solutions. Individuals who represent a single solution to a problem are mutually influenced during the search process, which leads to forming/canceling groups of individuals. Such groups organize themselves the movement of individuals, therefore the adjective self-organizing. Important advantage of this algorithm is based on its ability to process diverse data types of parameters like integers, real or discrete values.

After the initialization process that defines managing and finishing parameters, the population is created. Each individual is evaluated by a fitness function. The best individual is chosen for a leader in next migration cycle. After that all individuals start to move to the leader in steps. After each steps the individuals will be evaluated by the cost function. If the value of evaluation is better than the previous one it will be remembered. After each individual reaches the last step they return to new positions where the best fitness was found, i.e. beside the leader all the individuals will be moved to a new location. In other words they did searching in the state space and migrated. In such a manner one migration cycle has been finished and new one will immediately start after a new leader (the best individual) will be determined.

The movement is described by Eq. (8). 


$$
x_{i, j}^{M K+1}=x_{i, j, S T A R T}^{M K}+\left(x_{L, j}^{M K}-x_{i, j, S T A R T}^{M K}\right) * t * \text { PRTVector }
$$

where

$x_{i, j}^{M K+1}$ is value of $i$-individual of $j$-parameter in step $t$ in next migration step $M K+1$

$x_{i, j}^{M K}$ is value of $i$-individual of $j$-parameter in step $t$ in actual migration step $M K$

$x_{L, j}^{M K}$ is value of Leaders $j$-parameter in step $t$ in actual migration step $M K$

Parameter $t$ takes values from 0 to 1 by a defined step. The randomly generated binary perturbation vector PRTVector controls the allowed dimensions for an individual. If an element of the perturbation vector is set to zero, then the individual is not allowed to change its position in the corresponding dimension. The size of population remains the same, even the individuals are the same. The acquired knowledge remains in each individual and we can observe certain learning process during migration cycles. The only kind of selection can be observed in choosing the leader, which depends on the quality of an individual.

For optimization of ancillary services dispatch a single individual represents a single solution of the dispatch problem. i.e. time series of activated AS.

\section{SIMULATION RESULTS}

To test the optimization properties of the SOMA algorithm the proposed algorithm was applied to 2 case studies. At each sampling interval, the TSO control center had the following information:

- Prediction of ACE for the next 6 hours

- $\quad$ Prices and sizes of blocks that can provide ancillary services for the next 6 hours

The database of ancillary services that can be used for dispatch was described in Section 2. If two generating units provide the same ancillary service and have the same price they are replaced by a dummy source with size given by the sum of capacities of both sources to reduce the number of parameters.

The following parameter values to guide its search: population size of 100 and 50 migrations. The optimization time depends not on the number of parameters but on the computation time of the cost function. Depending on the step of the SOMA algorithm 100 - 500 computations of the cost function are needed for each generation.

At each sampling interval SOMA algorithm searches for solution that minimizes the cost function (4). This solution represents a time series of activated AS for next 6 hours with the least costs. Figure 4 shows the optimal solution obtained at the time 14:00 for the data from the first study. The area below green line given by the prediction of ACE represents the energy that lacks in the system and has to be obtained by activating the ancillary services. The secondary control reserve is operated automatically and should cover the fast component of the open-loop ACE below the line ACEuncovered since. The maximal power that can be used from the blocks that provide SR, QS, HV, TR are $300 \mathrm{MW}$, $600 \mathrm{MW}, 300 \mathrm{MW}$ and $170 \mathrm{MW}$ respectively. As in the predictive control strategy only the set-point changes to generating blocks that refers to the current sampling point are applied.

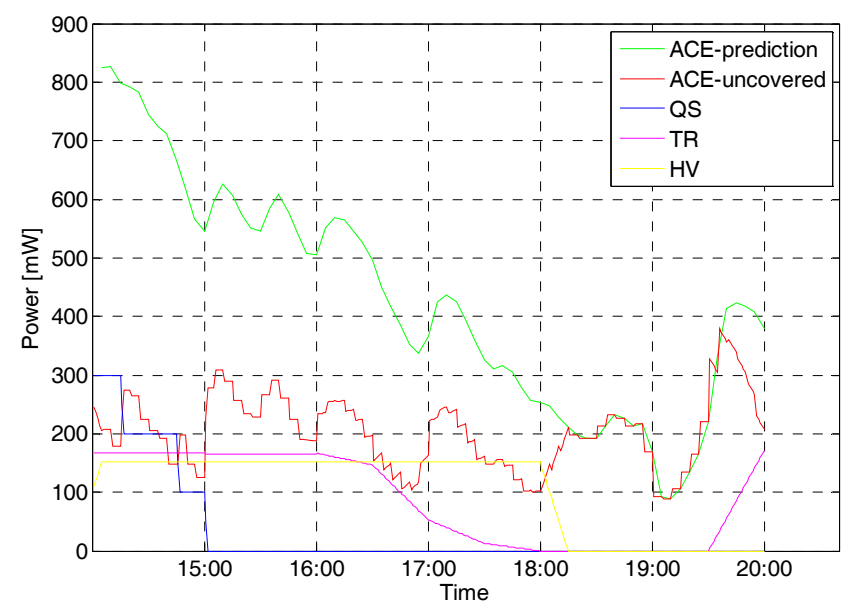

Fig. 4 Optimal activation of ancillary services at time 14:00

The value of cost function is shown in Figure 5. It can be seen that even during the population creation process one of individuals (solutions to the optimization problem) is close to the minimum. After 20 migrations the optimization is stopped and solution obtained. The computation time for a single optimization is a on Intel Pentium 3.0 GHz, 2GB RAM)

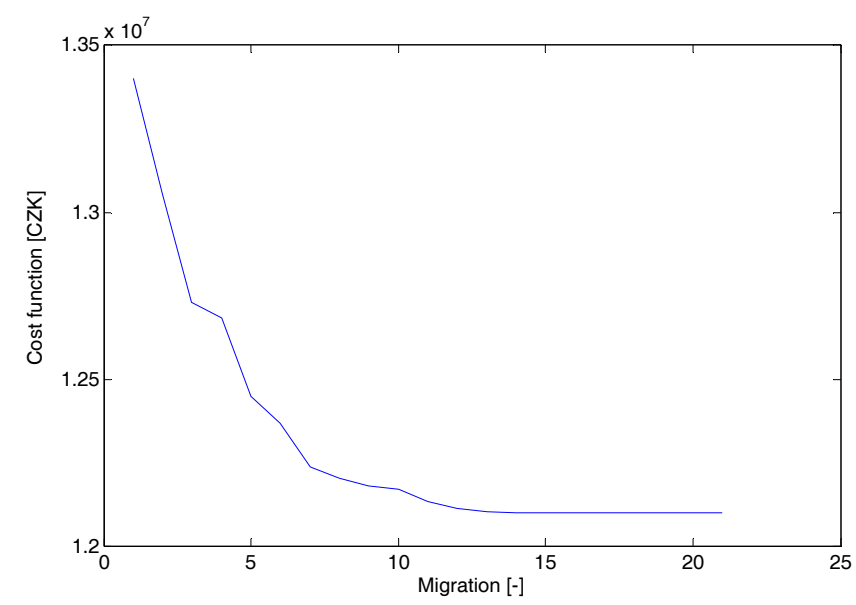

Fig. 5 Cost function history during the migration process

The first case study is for the short outage of a large generating unit. Figure 4 shows the course of ancillary services for the first study, where $d P_{o}$ represents open-loop ACE behaviour in undisturbed conditions. i.e. without any 
control and $d P_{c}$ represents the error between the production and consumption with ancillary services being applied (closed-loop). After the outage of a generating block is recognized in a system Quick-start reserve and Tertiary control are immediately activated. The undesired effect of counter-regulation occurred at time 14:00 caused by the discrepancy between the prediction and real value of $d P_{o}$.

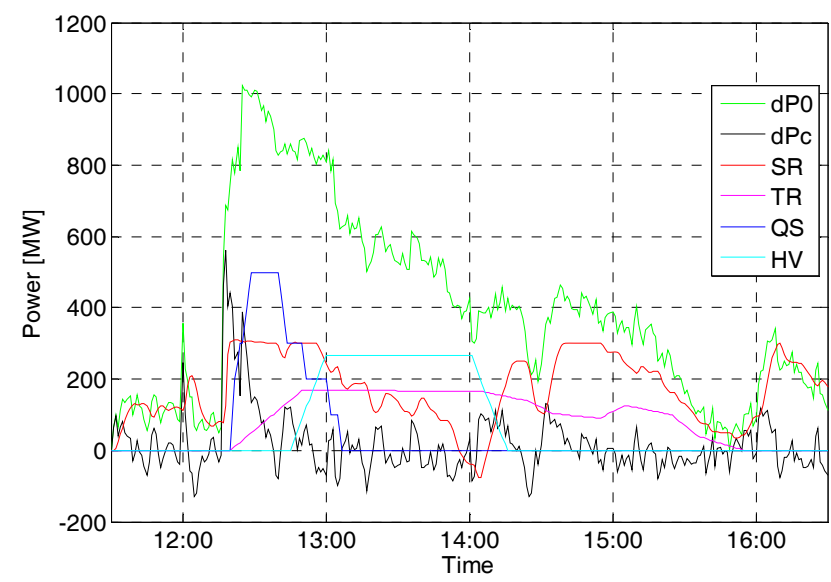

Fig. 6 Course of activation of ancillary services for the first case study

The second case study represents long-term lack of power in the power system. Using the ancillary services SR, TR, HV and QS the value of $d P_{o}$ is kept in the region around zero for minimum costs.

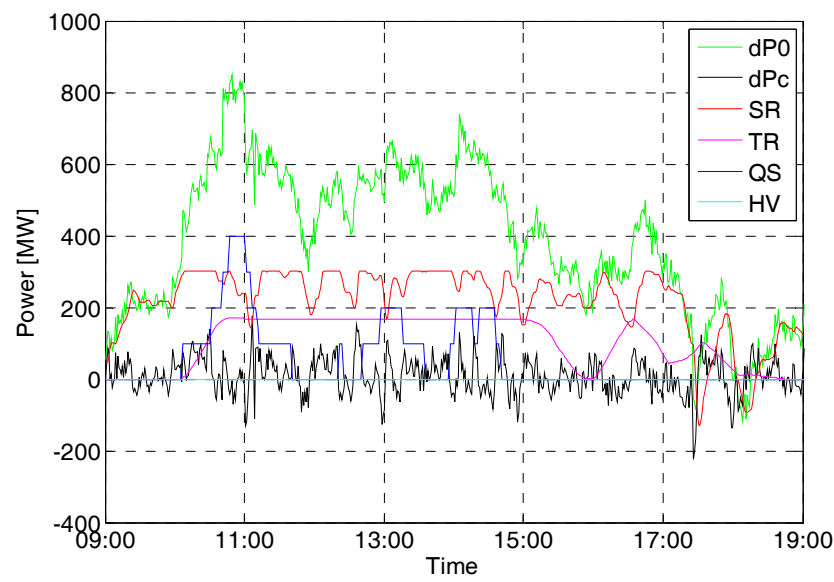

Fig. 7 Course of activation of ancillary services for the second case study

\section{CONCLUSIONS}

In the paper method for finding optimal activation of ancillary services using Self-Organizing Migration Algorithm is described. The proposed method for optimization of activation of ancillary services could help the TSO control center for training the human operators. The simulation results are promising and show the effectiveness of the proposed approach.

\section{REFERENCES}

Arnott, I., Chown, G., Lindstrom, K., Power, M., Bose, A., Gjerde, O., Morfill, R. and Singh, N. (2003). Frequency control practices in market environments. Proceedings of the Quality and Security of Electrical Power Delivery systems, 143-148.

Babu, B.V. and Onwubolu, G. (2004). New Optimization techniques in engineering. Springer-Verlag, England.

Chand, P. and Sugianto, L.F. (2005). Co-optimization of energy market and ancillary services. Proceedings of the Power Engineering Conference, 1001 - 1006.

Chuvychin, V.N., Gurov, N.S., Venkata, S.S. and Brown, R.E. (1996). An adaptive approach to load shedding and spinning reserve control during under frequency conditions. IEEE Transactions on Power Systems, 11, 1805-1810.

Damousis, I.G., Bakirtzis, A.G. and Dokopoulos, P.S. (2002). Network-constrained economic dispatch using realcoded genetic algorithm, IEEE Transactions on Power Systems, 18, 198-205.

Fan, J.Y. and Zhang, L. (1998). Real-time economic dispatch with line flow and emission constraints using quadratic programming, IEEE Transaction on Power Systems, 13, 320-325.

Havel, P., Horáček, P., Fantík, J. and Janeček, E. (2008a). Criteria for evaluation of power balance control performance in UCTE transmission grid. Proceedings of 17th IFAC World Congress, 6998-7003.

Havel, P., Horáček, P., Černý, V. and Fantík, J. (2008b). Optimal Planning of Ancillary Services for Reliable Power Balance Control. IEEE Transactions on Power Systems, 23, 1375-1382.

Schmitt, A. and Verstege, J.F. (2001). A multi-criteria optimization of ancillary services in a competitive energy market, Proceeding of IEEE Power Engineering Society Winter Meeting, 3, 1061 - 1066.

UCTE Operation Handbook (2004), v2.2/20.07.04, Union for the Co-ordination of Transmission of Electricity, http://www.ucte.org/ library/ohb/policy1 v22.pdf>.

Wang, X., Song Y.H. and Lu, Q. (2002). A coordinated real time optimal dispatch method for unbundled electricity markets, IEEE Transactions on Power Systems, 17, 482490.

Yoshida, H., Kawata, K., Fukuyama, Y., Takayama, S. and Nakanishi, Y. (2000) A particle swarm optimization for reactive power and voltage control considering voltage security assessment, IEEE Transactions on Power Systems, 15, 1232-1239. 